\title{
Balance de la investigación nacional sobre acción colectiva y movimientos sociales por la salud en Colombia*
}

\section{Balance of national research on collective action and social movements for health in Colombia}

\section{Pesquisa nacional sobre ação coletiva e movimentos sociais para a saúde na Colômbia}

Fecha de recepción: 21-11-13 Fecha de aceptación: 10-03-14

doi:10.11144/Javeriana.RGYPS13-26.bins

Yadira Eugenia Borrero-Ramírez**

María Esperanza Echeverry-López***

Este artículo corresponde a una investigación documental, tipo estado del arte, sobre la investigación nacional en acción colectiva y movimientos sociales en salud. Se desarrolló en el marco del proyecto Acción colectiva en salud en Colombia en el contexto de la reforma a la salud, 1994-2010, desarrollado entre 2010 y 2013 (fecha de iniciación: 27 de marzo de 2011. Fecha de terminación: 16 de septiembre de 2013), adscrito a la línea de investigación "Derecho a la Salud y Luchas Sociales por la Salud en Colombia" del Grupo de Investigación de Gestión y Políticas de Salud, Facultad Nacional de Salud Pública, Universidad de Antioquia (Colombia) y en el Grupo de Antropología Médica Crítica de la Universidad Nacional (Colombia) Código: 111552128282. Contrato RC № 309 de 2011, financiado por el Departamento Administrativo de Ciencia, Tecnología e Innovación de Colombia (Colciencias).

** Ph.D. en Salud Pública, magíster en Sociología. Directora del Departamento de Salud Pública y Epidemiología, Pontificia Universidad Javeriana Cali, Colombia. Dirección de correspondencia: Calle 18 No 118-250 Cali, Colombia. Correo electrónico: yborrero@javerianacali.edu.co

**** Ph.D en Salud Colectiva, magíster en Ciencias Políticas, magíster en Salud Pública. Docente titular Facultad Nacional de Salud Pública, Universidad de Antioquia, Medellín (Colombia).Correo electrónico esperanzaecheverry@yahoo. com.mx 


\section{Resumen}

Objetivo: este artículo presenta el balance nacional de los estudios relacionados con la acción colectiva y los movimientos sociales por la salud en Colombia. Método: se realizó un estado del arte que incluyó la revisión de investigaciones de dos fuentes: tesis de posgrado elaboradas entre 1990 y 2012 y artículos científicos publicados en las bases de datos de revistas indexadas en este mismo periodo. Resultados: los resultados mostraron que hay un predominio de investigaciones en clave de contienda sociopolítica así como la existencia de algunas investigaciones emergentes que analizan propiamente la acción colectiva y/o los actores que luchan por la salud. Conclusión: el campo de la investigación sobre la acción colectiva por la salud es incipiente a nivel nacional, los enfoques teóricos dominantes son estructuralistas, y aún hay pocas investigaciones que se aproximen al estudio de los procesos de configuración de actores colectivos subalternos que disputan el campo sanitario.

Palabras clave: reforma de la atención de salud; política de salud; ciencias sociales; salud pública; derecho a la salud; participación social

Palabras clave descriptor: reforma de la atención de la salud; política de salud; ciencias sociales; salud pública; derecho a la salud; participación social

\section{Abstract}

Objective: The aim of this article is to present the balance of national studies related to collective action and social movements for health in Colombia. Method: A state of the art, that included the revision of research from two sources: thesis conducted between 1990 and 2012 and research articles published in databases from indexed journals in the same period. Results: The results showed that research has been more predominant specifically regarding sociopolitical struggles and the existence of some emergent research that analyze collective action and/or the actors who struggle for health. Conclusion: The field of the research on the collective action for health is emerging at national level, the theoretical approaches are mostly structuralists, and there are still few investigations that approximate the study of the processes of configuration of secondary collective actors that dispute the health field.

Keywords: health care reform; health policy; social sciences; right to health; public health; social participation

Keywords plus: health care reform; health policy; social science; public health; right to health; social participation

\section{Resumo}

Objetivo: Este artigo apresenta o balance nacional dos estudos relacionados com a ação coletiva e os movimentos sociais pela saúde em Colômbia. Método: Foi realizado um estado da arte que incluiu a revisão de pesquisas de duas fontes: teses de pós-graduação elaboradas entre 1990 e 2012 e artigos científicos publicados nas bases de dados de revistas indexadas neste mesmo período. Resultados: Os resultados mostram que existe um predomínio de pesquisas em chave de contenda sociopolítica assim como a existência de algumas pesquisas emergentes que analisam propriamente a ação coletiva e/ou os autores que lutam pela saúde. Conclusões: O campo da pesquisa sobre a ação coletiva pela saúde é incipiente a nível nacional, os enfoques teóricos dominantes são estruturalistas, e ainda a poucas pesquisas que aproximam-se ao estudo dos processos de configuração de atores coletivos subalternos que disputam o campo sanitário.

Palavras chave: Reforma dos serviços de saúde; política de saúde; ciências sociais; saúde pública; direito à saúde; participação social

Palavras chave descritores: reforma dos serviços de saúde; política de saúde; ciências sociais; saúde pública; direito à saúde; participação social 


\section{Introducción}

En Colombia a partir del año 2010 se percibe una intensificación de la acción colectiva por la salud (AC), vinculada inicialmente con la declaración de la llamada "emergencia social"1 durante el gobierno de presidente Álvaro Uribe Vélez. Pese a la posterior derogatoria de ese intento de reforma, por parte de la Corte Constitucional, desde entonces la conflictividad en el campo de la salud -expresada en protestas sociales y emergencia de movimientos sociales por la salud- se ha mantenido presente e involucró el debate legislativo sobre la reforma en salud, desarrollado durante el año 2013. La contienda ha utilizado repertorios contenciosos -como marchas, plantones, tomas, entre otrosy no contenciosos, como la participación del movimiento social por la salud en los debates del Congreso de la República.

La persistencia de esta conflictividad social en el campo de la salud condujo a preguntarse por la dinámica de la AC y la existencia [o no] de movimientos sociales (Ms) durante el proceso de reforma sectorial en Colombia. Un punto de partida necesario fue el asunto que orientó este trabajo: ¿qué se ha investigado en el país alrededor de la acción colectiva y los movimientos sociales por la salud en el marco de la reforma? Tres razones motivaron esa pregunta: la persistencia de malestar social en el campo de la salud, evidente ante la presencia de protestas sociales y su subsecuente visibilidad a través de los medios de comunicación nacional; la posibilidad de anidar la investigación en una línea y en un proyecto más general, orientado a conocer

1 La emergencia social es una figura de excepción contemplada en la Constitución Política (junto con el estado de guerra exterior y el estado de conmoción interior), específicamente en el artículo 215, que considera que esta se puede declarar cuando "sobrevengan hechos [...] que perturben o amenacen perturbar en forma grave e inminente el orden económico, social y ecológico del país, o que constituyan grave calamidad pública...”. cómo había sido la AC por la salud durante el periodo de reforma; y el hecho de que se requería un estado del arte que permitiera conocer cómo se han estudiado estos fenómenos en el país, para avanzar teórica y metodológicamente en la línea propuesta.

El estado del arte tuvo tres objetivos: primero, conocer las investigaciones realizadas en $\mathrm{Co}$ lombia sobre AC y Ms por la salud desde 1994, momento en que se empieza a implementar la reforma; segundo, identificar cuáles eran las tendencias teóricas y metodológicas que habían estado presentes en estas investigaciones; y, finalmente, definir vacíos en la investigación nacional alrededor de este nuevo problema.

El contexto de esta pregunta es un campo interdisciplinario de investigación, que ha ganado mayor visibilidad en las tres últimas décadas: el estudio de la acción colectiva y los movimientos sociales; en él confluyen aportes de disciplinas como historia, sociología, antropología y ciencias políticas. Además, puede construir un diálogo con las preocupaciones propias de la salud pública alrededor de cómo se constituyen los sistemas de salud y el derecho a la salud.

Este trabajo correspondió a un estado del arte nacional -comprendido como un balance crítico de la investigación- alrededor de la producción académica sobre AC y MS por la salud. Para su desarrollo se diseñó un análisis documental, a través del cual se revisó la producción académica vinculada al tema, con el objetivo de conocer tendencias, orientación, avances y preguntas o vacíos en dicha producción. La búsqueda incluyó tesis de maestría y doctorado, artículos relacionados en bases de datos de revistas indexadas, artículos sobre análisis de coyuntura de la protesta social y revisión de otra producción académica -libros y capítulos de libro-relacionada con el tema. En total se incluyeron 27 trabajos. 
Las definiciones sobre AC y MS presentan amplios matices de acuerdo a las perspectivas teóricas y epistemológicas en que se inscriben (1); sin embargo, y pese a ello, podemos afirmar que la AC comparte algunos elementos centrales que ayudan a su comprensión y corresponde a: “... una experiencia compartida de 'malestar social' vinculada a una necesidad no resuelta en los ámbitos público o privado y/o a una percepción de injusticia; se expresa a través de protestas o luchas sociales y de movimientos sociales (MS), estos últimos con cierta permanencia en el tiempo" (2). Tal como lo plantea Archila (3), las AC y los Ms evidencian conflictos no resueltos en la sociedad.

Las perspectivas teóricas para explicar la ACy los Ms han transitado un amplio espectro conceptual. Inicialmente, fueron comprendidas como expresiones de la acción irracional de las masas (4); con posterioridad, y desde las contribuciones de Olson (5), se introdujo el análisis costo-beneficio que orienta la acción colectiva, aportando un elemento central: la AC no es irracional (6) y los procesos de investigación tienen el reto de comprender y develar esa racionalidad. Esta idea ha propiciado un amplio debate teórico y metodológico en ese campo de estudio.

Pese a la cantidad y complejidad de enfoques sobre el tema y los avances de la segunda mitad del siglo xx, aún no se supera completamente la dicotomía sujeto-estructura. Las propuestas teóricas configuraron dos paradigmas dominantes desde la década de los ochenta, las cuales pueden clasificarse -de manera genérica- en perspectivas que priorizan el análisis desde las condiciones estructurales y aquellas que privilegian el estudio desde los actores. ${ }^{2}$ Sin embargo, vale

2 En una publicación anterior (2) se presentó de una manera amplia el debate conceptual de este tipo de problemas de investigación y se avanzó en plantear una propuesta teórica para abordar la la pena llamar la atención en que además de estos enfoques son claves los aportes del marxismo, las teorías de la resistencia y recientemente las teorías de enmarcación (2).

Para el caso de esta investigación se presenta un análisis de los trabajos desarrollados en este campo en Colombia, clasificados en cuatro grupos: 1) estudios clásicos y pioneros de la Ac y los Ms en Colombia; 2) investigaciones sobre proceso, actores y configuraciones de poder en la formulación de políticas de salud previos a la reforma; 3 ) estudios orientados a comprender la reforma a la salud como un escenario conflictivo de disputa. Aquí se hallaron dos perspectivas: la estructuralista y las de los actores; 4) análisis de coyuntura sobre la protesta social en general y la incidencia de las luchas por la salud en ese contexto.

\section{Método}

\section{Tipo de estudio}

Este estudio correspondió a un estado del arte, llamado también "estado del conocimiento", que acá se concreta en una revisión crítica de literatura. Es considerado un tipo de investigación documental, que de manera sistemática, analítica y crítica reflexiona sobre el conocimiento construido alrededor de un asunto específico, en este caso sobre las investigaciones desarrolladas en el país cuya temática central fue la acción colectiva por la salud y las contiendas en el campo

investigación alrededor de las luchas por la salud. Dentro de los cuerpos teóricos que buscan entender la acción colectiva desde las estructuras políticas, están la movilización de recursos, la estructura de oportunidades políticas y la contienda política. Otros enfoques privilegian la perspectiva de los actores: teorías de la identidad; el construccionismo, y los marcos de sentido. Otras teorías como las de la resistencia y del feminismo crítico, a nuestro juicio, aportan elementos teóricos para comprender las luchas, con frecuencia invisibles, de los actores subalternos. 
sanitario. Es decir, el estado del arte como propuesta investigativa implica la realización de un balance crítico sobre el tema. Como afirman Galeano y Vélez (7): “es una investigación sobre la producción investigativa, teórica o metodológica -existente acerca de un determinado tema- para develar la dinámica y la lógica presentes en la descripción o interpretación que del fenómeno en cuestión hacen los teóricos o investigadores". Esta investigación fue aprobada por el Comité de Ética de la Facultad Nacional de Salud Pública de la Universidad de Antioquia, en el marco de una tesis doctoral anidada.

Para responder la pregunta de investigación, se realizó análisis documental, entendido este como una estrategia de investigación social y no exclusivamente como una técnica útil para la recolección y el procesamiento de información (8). Esto implicó un proceso de selección de fuentes, análisis crítico de estas y presentación de resultados. La búsqueda involucró el periodo 1990-2012.

\section{Fuentes de información}

El análisis documental incluyó la revisión de las investigaciones que se habían desarrollado sobre acción colectiva y movimientos sociales por la salud en el país, a partir de dos fuentes iniciales: 1) la búsqueda y revisión de las tesis elaboradas entre los años 1990 y 2012 sobre el tema por estudiantes de posgrado -maestría y doctorado- de programas en salud pública, sociología, historia, antropología y ciencias políticas, y 2) la búsqueda y revisión de artículos científicos sobre el tema en las bases de datos de revistas indexadas en Scielo, Academic OneFile, Ebsco-Fte académica, oCDE y Science Direct. Los buscadores utilizados en español e inglés fueron: acción colectiva y salud, luchas por la salud, struggles for health y collective action for health.
La primera etapa de búsqueda encontró principalmente investigaciones sobre participación en salud y estudios sobre la reforma colombiana, y halló solo dos tesis de doctorado con resultados específicos sobre acción colectiva por la salud y un artículo en revista indexada sobre movimientos sociales por la salud. Una vez agotada esta revisión se complementó con otras dos fuentes: 3) la pesquisa de producción académica -tipo libros o capítulos de libro-, centrada en el análisis de los procesos de reforma en salud que incluyeran el registro de los actores involucrados; 4) las protestas sociales -por todas las causas-, en Colombia, a través de la revisión de 25 artículos de la revista Cien Días entre 1994-2009, ${ }^{3}$ para conocer el lugar que ocupaba la salud como eje de movilización social.

Los criterios de inclusión de las fuentes documentales halladas fueron: a) trabajos representativos ${ }^{4}$ sobre movimientos sociales (MS) y acción colectiva (AC) en Colombia, en general, que mostraran las grandes tendencias académicas sobre este campo de investigación en el país; b) investigaciones sobre política de salud y/o derecho a la salud en perspectiva sociohistórica; c) trabajos específicos sobre acción colectiva en salud; d) análisis de coyuntura sobre la protesta social. Los dos primeros criterios fueron útiles para

3 La revista Cien Días, es como su nombre lo indica, una publicación periódica del Centro de Investigación y Educación Popular (Cinep), que contiene una sección sobre protestas sociales - por todas las causas- en el país, elaborada a partir del seguimiento diario de la prensa nacional y regional. Estos artículos fueron analizados siguiendo las categorías de actores, motivos, repertorios, adversarios de esas luchas y respuesta gubernamental. Con esa información se diseñó una base de datos cualitativa, cuyos resultados se sistematizaron en una publicación anterior (véase el trabajo de Borrero y Echeverry (2), referenciado en la bibliografía de este artículo).

4 El criterio de representatividad se estableció por la tradición y el reconocimiento académico de autores colombianos en este campo, trabajos revisados en el curso de un seminario realizado en la Universidad Nacional en 2009 por una de las autoras de este artículo. 
conocer la configuración histórica del campo sociosanitario como un contexto necesario para el tema estudiado. Los otros dos criterios apuntaron a responder concretamente la pregunta de investigación documental: el estado del arte de la investigación sobre AC y Ms, y a conocer la incidencia de la salud en el conjunto de la protesta social. Se excluyeron los trabajos sobre participación social y análisis jurídico-normativo, económico o financiero de la reforma a la salud porque no aportaban al objetivo de este estudio. De todo este proceso resultaron 27 trabajos que revisar.

\section{Análisis de información}

El análisis documental dialogó en forma permanente con el instrumento elaborado para la revisión de los trabajos seleccionados. Inicialmente, se diseñó una guía que se fue ajustando con la incorporación de nuevas líneas analíticas que emergieron en el proceso de sistematización. Así, en la revisión de los 27 trabajos seleccionados se tuvieron en cuenta: a) el periodo estudiado; b) el enfoque teórico; c) los objetivos y la metodología; d) las perspectivas y/o configuraciones históricas sobre el objeto de estudio -por ejemplo: la política de salud, el derecho a la salud-; e) pervivencias, rupturas y coyunturas de cambio en esas trayectorias históricas; f) identificación de los actores participantes en los procesos estudiados, así como de sus propuestas o reivindicaciones; g) configuración -distribución y asimetría- de las relaciones de poder; h) balance-incidencia, resultados y/o conclusiones- ${ }^{5}$

El análisis aportó las categorías que guiaron el ordenamiento de los resultados, agrupando la revisión en cuatro tipos de estudios, que se

5 Los trabajos revisados no incorporaban todas las líneas analíticas que se presentan aquí. Una guía busca incluir amplias posibilidades de sistematización aunque las fuentes sean heterogéneas. presentan a continuación: 1) trabajos orientados a la comprensión histórica de grandes Ms en el país, diferentes a los de salud; 2) investigaciones sobre proceso, actores y configuraciones de poder en la formulación de políticas de salud; 3) estudios orientados a comprender la reforma a la salud como un escenario conflictivo de disputa. Aquí se hallaron dos perspectivas: las estructuralistas y las de los actores; 4) análisis de coyuntura sobre la protesta social en general y la incidencia de las luchas por la salud en ese contexto.

\section{Resultados}

1. Los estudios pioneros sobre acción colectiva y movimientos sociales en Colombia: preocupaciones diferentes a la salud

En esta categoría se revisaron 8 trabajos orientados al estudio de actores y Ms más tradicionales y/o visibles. Una síntesis de los aspectos más relevantes muestra que en Colombia el problema de las luchas sociales, la acción colectiva y los movimientos sociales ha sido planteado ampliamente por los científicos sociales. Algunos de los principales investigadores en este campo han centrado la atención en la comprensión de los procesos de configuración de actores colectivos, como la formación de la clase obrera, del movimiento popular, del movimiento afrodescendiente y de los movimientos cívicos. Entre los investigadores más reconocidos por sus estudios alrededor de los procesos de lucha en Colombia están Archila $(9,10)$, Escobar y Pedroza (11) y Múnera (1), quienes han aportado al conocimiento de las luchas de la clase obrera, del movimiento estudiantil, del movimiento afrodescendiente y de las luchas cívicas, entre otros.

Desde la noción de resistencia se han hecho varias investigaciones sobre el movimiento indígena, campesino y afrodescendiente 
$(12,13)$-la producción académica más abundante relacionada con este enfoque ha sido alrededor del movimiento indígena y su historia- y la resistencia contra la violencia armada en Colombia $(12,14,15)$. Sin embargo, el análisis específico sobre las luchas por la salud en el país es un asunto más reciente en la investigación nacional.

Este primer grupo de trabajos fue útil para conocer los antecedentes y los énfasis de la investigación sobre AC en el país, la importancia del concepto de resistencia para una comprensión integral de los Ms, y también para constatar que la organización y movilización por la salud en el país no estuvo, hasta hace una década, en las preocupaciones académicas en este campo.

\section{Investigaciones en clave histórica y/o} sociopolítica acerca del debate sobre las políticas de salud antes da la reforma de los noventa

Aquí se inscriben 7 estudios orientados a comprender, desde el enfoque sociopolítico o desde la perspectiva histórica, cómo diferentes actores han disputado el campo sanitario. ${ }^{6}$ La mayoría ha leído el problema en clave estructural, inscrito en el debate que sobre las políticas de salud se desarro-

6 Se entiende la noción de campo en la perspectiva de Bourdieu, quien plantea: "pensar en términos de campo es pensar en términos de relaciones" (16). Así, el campo: "puede definirse como una red o configuración de relaciones objetivas entre posiciones. Estas posiciones se definen objetivamente en su existencia y en las determinaciones que imponen a sus ocupantes, ya sean agentes o instituciones, por su situación (situs) actual y potencial en la estructura de la distribución de las diferentes especies de poder (o de capital) -cuya posesión implica el acceso a las ganancias específicas que están en juego dentro del campo-y, de paso, por sus relaciones específicas con las demás posiciones (dominación, subordinación, homología, etc.) [...] microcosmos sociales relativamente autónomos, espacios de relaciones objetivas, que son irreductibles a las que rigen los demás campos". lla dentro del Estado. En este campo se ha demostrado la complejidad de actores e intereses involucrados en el proceso de toma de decisiones en salud, así como los recursos diferenciales de poder y la incidencia de los actores en la política pública (17-20).

\section{Los trabajos de Hernández y Quevedo} muestran, en clave histórica e historiográfica, cómo diferentes sectores y fuerzas sociales han participado e incidido en los debates y en las decisiones de políticas de salud, con especial énfasis en la creación del Sistema Nacional de Salud, del Seguro Social y en el proceso de reforma de 1993 (20). Su propuesta teórica se ha construido con base en las corrientes del institucionalismo histórico ${ }^{7}$ y los aportes de Michael Mann para interpretar "las redes de poder", la constitución de actores sociales más allá de la noción de clase social y las relaciones conflictivas entre aquellos. Una de sus conclusiones más relevantes para la investigación sobre luchas por la salud es sin duda la configuración histórica de los actores sociales en salud y la pervivencia de la fragmentación como una característica de los sistemas de salud segmentados, producto, entre otras razones, de la primacía de intereses corporativos particulares sobre el interés general y de las diferencias de poder que esos actores movilizan en la disputa por el campo sanitario.

Asimismo, estos estudios evidencian cuáles fueron los principales actores sociopolíticos que participaron de los debates alrededor de la formulación e implementación de las políticas de salud (17-19). En los primeros periodos, especialmente durante la llamada hegemonía conservadora, los actores identi-

7 Institucionalismo histórico: analiza las configuraciones institucionales más que escenarios particulares. Hay una tendencia a interpretar los procesos históricos de largo plazo y las coyunturas específicas, y toma contextos macro para encontrar claves explicativas. 
ficados más importantes fueron los médicos, quienes promovían la participación del Estado en la higiene pública centrada en el control de las epidemias. Posteriormente, durante el periodo de la República Liberal, fue muy visible la participación del emergente asalariado urbano, a través de los sindicatos que se movilizaron alrededor de reivindicaciones por la regulación de la jornada laboral y la conformación de un sistema de salud que los protegiera de los riesgos. Gómez (21) llama la atención desde la noción teórica de grupos de interés, sobre la presencia de actores similares en la discusión política del sistema de salud colombiano.

Es claro en este conjunto de investigaciones que hubo por lo menos dos motores que impulsaron la "modernización” del país alrededor de las políticas de salud. De un lado, la necesidad de mejorar las condiciones para participar en el comercio internacional, lo que implicaba controlar el riesgo de epidemias en los puertos y mejorar la productividad de la mano de obra (17). En este sentido desempeñaron un papel muy importante las recomendaciones del Gobierno de los Estados Unidos, a través de la Fundación Rockefeller, para implementar campañas como el control de la uncinariasis en la zona cafetera. De otro lado, las investigaciones muestran la incidencia que logran las presiones internacionales para orientar la política de salud y de los sistemas de salud $(17,22)$.

Pese a esto, las investigaciones también han sido contundentes al demostrar que la salud no ha sido considerada por el Estado colombiano como un derecho de ciudadanía (2325) y, en contraste, se ha institucionalizado un sistema de salud "fracturado", como lo denomina Hernández, que reproduce en su interior las inequidades sociales propias de la pertenencia de clase. Sin embargo, estos análisis no se preguntan por las AC en salud ni por los procesos de configuración de actores sociales subalternos.

\section{Estudios sobre la reforma a la salud como un escenario de disputa y configuración de actores colectivos en Colombia}

La revisión realizada encontró 12 estudios de diferente alcance que buscan comprender, desde el 2005, la Ac por la salud en Colombia; seis de ellos desde enfoques estructuralistas y seis más desde la perspectiva de la agencia, es decir, de los actores.

Desde el año 2005 y a partir de la revisión de artículos y de tesis de posgrado en salud pública y otras áreas afines, se encuentra que emerge un interés creciente de la Academia colombiana por analizar algunas de las luchas por la salud llevadas a cabo a partir de la promulgación de la Ley 100 de 1993. En este sentido, se encuentran dos enfoques predominantes: el primero sigue la línea teórica de Hernández alrededor de las perspectivas estructuralistas para el análisis de la política de salud, pero involucra la noción de AC, específicamente desde los enfoques de la estructura de oportunidades políticas y la contienda entre actores sociopolíticos. El segundo, por su parte, corresponde a un pequeño conjunto de investigaciones cuyos esfuerzos se concentran en mirar los actores sociales que disputan en el campo sanitario.

\section{Disputas en el campo de la salud: aproxi- maciones desde enfoques estructuralistas.} Desde las perspectivas estructuralistas de la AC y los Ms, recientemente encontramos un pequeño grupo de trabajos inaugurados por dos tesis. El primero, una tesis de maestría en sociología de una de las autoras (26), en la cual con un enfoque teórico neomarxista se intenta mostrar que la discusión que se dio durante la reforma de 1993 vinculaba 
a los diferentes actores y sus técnicos ${ }^{8}$ a intereses de clases específicos, así como la debilidad organizativa y de recursos de actores potencialmente antagónicos a la reforma propuesta por el gobierno -algunas facciones sindicales-y, finalmente, encuentra en la formulación de la Ley 100 de 1993 procesos corporativistas de negociación política.

La segunda corresponde a la tesis doctoral de Mónica Uribe (27). Allí se reconstruyó el proceso de contienda política alrededor de la promulgación e implementación de la Ley 100 de 1993, identificando los actores sociopolíticos participantes, destacando la emergencia de nuevos actores en la última década del siglo xx como el Movimiento Nacional por la Salud y la Seguridad Social (MNSss) y el movimiento indígena, pero no se detiene específicamente en el análisis de estos actores ni es su interés central.

A estos estudios se suman más adelante la tesis de pregrado de Dayan Ocampo (28) y la de maestría de Mauricio Torres (29). Ambas abordan específicamente el estudio de la AC contenciosa ${ }^{9}$ por la salud. La primera, en relación con la crisis hospitalaria durante el periodo de la reforma, acude a la perspectiva teórica de la estructura de oportunidades políticas y muestra que en dicho periodo las AC han sido permanentes, con tres ciclos de intensificación de la protesta. Los

8 Se denominan técnicos a profesionales de diferentes disciplinas -salud, económicas, jurídicas- cuyos aportes al debate se hicieron privilegiando argumentos y lenguajes técnicos. Se encontraban vinculados a los gremios económicos y/o a estructuras formales del gobierno -Ministerio de Salud, de Trabajo y Planeación Nacional-, y en algunos casos al Seguro Social (institución desde la cual se hizo amplia oposición a la Ley 100).

9 La AC contenciosa hace referencia a la utilización, por parte de actores sociales con menos poder o subalternos, de repertorios de acción no institucionalizados como marchas, plantones, tomas, cierre de vía. Corresponde a una estrategia para la disputa en el campo sanitario. trabajadores de la salud son sus principales organizadores, pero se han ido sumando estudiantes y pobladores urbanos. Las demandas transitaron de asuntos salariales de tipo gremial y se politizaron a la disputa por el derecho a la salud. La respuesta estatal y paraestatal dominante ha sido la represión política (28).

La investigación de Torres (29) corresponde a un estudio de caso de las AC contenciosas y no contenciosas ${ }^{10}$ en Bogotá durante el periodo de la reforma (1994-2010). Para ello sigue el enfoque de contienda política y encuentra que las AC en dicha ciudad han sido constantes pero no lograron configurar ciclos de protesta. Sin embargo, reconoce que el campo sanitario se ha convertido en un escenario de contienda. Al igual que la investigación anterior, halló que los sindicatos son actores centrales de la contienda y con el tiempo se han ido sumando estudiantes y pobladores, entre otros. Asimismo, plantea que localmente no se ha logrado construir una identidad colectiva sólida ni revertir las tendencias estructurales de la política de salud (29).

Además, siguiendo la misma línea argumentativa, las tesis de doctorado de Carolina Morales Borrero y Zulma Urrego $(30,31)$ se suman a los intereses académicos por comprender las disputas en el campo de la salud. Morales Borrero (30), desde el enfoque de la contienda política, analiza la disputa entre actores sociopolíticos respecto a la formulación de las políticas de salud sexual y reproductiva (SSR) para jóvenes durante el periodo 1992-2005.

10 La AC no contenciosa corresponde al uso de canales institucionales para incidir en la disputa desarrollada en el campo de la salud. Ejemplo de ello son las reuniones de asociaciones de usuarios, asambleas de usuarios de la salud, etc. 
El principal aporte de ese enfoque es su contribución al esclarecimiento de los cambios en las políticas, de sus trayectorias y de las relaciones con las acciones colectivas y los repertorios utilizados. La investigación encontró que las políticas de SsR tuvieron una inflexión en 1992, como consecuencia del agotamiento del modelo cepalino, y en el marco del Consenso de Washington el énfasis dominante fue preventivista. Para 2005 encontró que esa orientación es cuestionada, gracias al impulso de los movimientos de mujeres y feminista. La investigación logra mostrar las tensiones en la formulación de las políticas públicas, pero no ahonda en los procesos de configuración de los actores de la contienda (30).

Finalmente, la investigación de Urrego (31), desde una mirada del proceso político y del papel de la cultura en la configuración de la AC con relación a las enfermedades transmitidas por vectores ${ }^{11}$ (ETV), analiza la disputa sostenida en este campo. El estudio muestra, de un lado, las limitaciones de las teorías de AC de Tilly y Tarrow para comprender las AC contenciosas por ETV en el país, especialmente ante la paradoja de que en este campo específico las AC no se incrementan en los típicos ciclos de protesta, sino -coincidiendo con la investigación de Ocampo (28) - en periodos de fuerte represión política y cierre del régimen político. Y de otro lado, que el desarrollo de AC por ETV ha requerido una fuerte activación de procesos sociales, articulación y existencia de redes de actores. La conclusión es que esto demuestra la existencia de ms por la salud, lo cual contrasta con posturas dominantes a nivel nacional que plantean la inexistencia de verdaderos Ms.

11 Las ETV son aquellas patologías que se presentan en la población y son causadas por la transmisión del agente causal o patológico a través de otro organismo que actúa como transmisor. Un ejemplo de ello es la malaria transmitida por el Anopheles.
Disputas en el campo de la salud: un giro de la mirada hacia los actores. Dentro del grupo de investigaciones que centran la mirada en los actores, la primera referencia que se encontró sobre luchas por la salud en Colombia fue un estudio etnográfico de tipo descriptivo sobre los procesos de resistencia de los trabajadores del hospital San Juan de Dios de Bogotá. Esta investigación no avanza específicamente en reflexiones teóricas sobre las resistencias, pero tiene el enorme valor de ubicar en el escenario los discursos de los trabajadores (32). Asimismo, la primera investigación que encontramos sobre otros actores diferentes a los trabajadores organizados corresponde al trabajo de Cristina Escobar y Gustavo De Roux (33), en el marco de un análisis sobre movimientos populares en Colombia entre 1970-1983, el cual documentó procesos organizativos y reivindicativos específicos por la salud vinculados a comunidades eclesiales de base en zonas de difícil acceso, como el departamento de Caquetá, y en sectores urbano-marginales, y también en las luchas cívicas de los setenta, las cuales incluían demandas por salud. Sin embargo la mención no profundiza el tema.

Un ejercicio inicial para comprender un actor colectivo fue el realizado por Karol Cotes (34), quien indaga sobre el proceso de construcción de la Federación de Asociaciones de Usuarios de Servicios de Salud (Fausalud), desde enfoques de participación y democracia. Logra mostrar que tal configuración es un proceso complejo, con tensiones internas, y que también enfrenta las restricciones del contexto social y político nacional, pese a lo cual la interacción con algunos actores claves emergentes en el actual sGsss va generando un proceso de politización de la organización y una apropiación de la lucha por la salud en clave de derecho.

En esta línea argumentativa se añaden dos investigaciones que además incursionan 
teóricamente en la noción de resistencia. De un lado, Esperanza Echeverry (35) inaugura un importante esfuerzo por comprender las lógicas de resistencia que nacen en el corazón de los ciudadanos y ciudadanas que participan en las organizaciones de usuarios de la salud (ous) y que han contribuido a la construcción del derecho a la salud. Como sostiene la autora:

\begin{abstract}
Los usuarios han contribuido a la construcción del derecho a la salud a través de la acción colectiva, haciendo visibles los asuntos críticos o componentes no realizados de ese derecho, ampliando la esfera pública con la pluralidad organizativa y la emergencia de nuevos actores y demandas y, construyendo redes de apoyo, a través de las ous, para mediar con las instancias del sGsss y del gobierno local en la realización del derecho a la salud (35).
\end{abstract}

En este mismo sentido, los investigadores Cesar Abadía, Diana Oviedo y Adriana Martínez (36) hacen una reflexión alrededor de una experiencia de resistencia a través de una organización social de Bogotá: Salud al Derecho. Sin embargo, estos esfuerzos analíticos no avanzan prioritariamente en el análisis de los procesos de configuración de actores colectivos ni en el marco de sentido para la acción.

La última investigación hallada en esta perspectiva corresponde a la tesis de pregrado de Liliana García y Narayana Betancur (37), en la cual se reconstruye el proceso de organización del "Martes por la Salud", repertorio de resistencia pacífica que se ha desarrollado en Medellín desde febrero de 2010. El esfuerzo analítico se realizó desde la perspectiva constructivista de Melucci. En este sentido, corresponde a un enfoque novedoso en la investigación nacional al86 rededor de la disputa por la salud. En este se da un lugar relevante a la comprensión procesual de los actores y de los movimientos sociales, aportando categorías claves como historicidad de los actores, redes sumergidas y procesos de sociabilidad, entre otros.

4. El análisis de coyuntura: la salud, un nuevo eje de movilización en el conjunto de la protesta social

Además de las investigaciones históricas y de aquellas centradas en los actores, y ante la ausencia para la mayor parte del periodo indagado de investigaciones específicas sobre AC y MS por la salud en Colombia, una revisión sistemática de la revista Cien Días (2) mostró que las luchas sociales por la salud son más invisibles que inexistentes durante la reforma; que han persistido durante todo el periodo estudiado (1994-2009) y que usualmente se articulan a otras luchas como la defensa del medio ambiente, el acceso a servicios públicos, la defensa del derecho al trabajo, el mejoramiento de las condiciones de vida a nivel local, las reivindicaciones de los pueblos indígenas, las luchas cívicas y las luchas de las mujeres por la autonomía.

Asimismo, las demandas más visibles han sido contra la privatización de la red pública hospitalaria y por la crisis -cierres, despidos, reestructuraciones- de estas instituciones, por el acceso a servicios de salud y a la seguridad social, y más recientemente, este conjunto de demandas se politizan y son nombradas como derecho. Además, hay presencia de diversos actores como sindicalistas, estudiantes, usuarios, pacientes y trabajadores del sector. Durante los últimos años emergen nuevos actores como las madres comunitarias, la población recluida en cárceles y en situación de desplazamiento, así como procesos de articulación entre actores.

Sin embargo, estas investigaciones, producto de análisis sistemático de la coyuntura nacio- 
nal, no han ubicado como elemento central de la reflexión la protesta en salud y tampoco la comprensión de los procesos de configuración de actores colectivos subalternos en el campo sanitario (2).

\section{Discusión}

Este estudio tuvo como objetivo realizar un balance de la investigación nacional sobre acción colectiva y movimientos sociales por la salud, con el objeto de identificar las perspectivas teóricas y metodológicas que orientaron los trabajos, así como comprender cómo se han constituido las contiendas en el campo de la salud como objeto de investigación y develar las tendencias, actores y recursos de poder.

Los principales resultados indican que la preocupación académica por investigar las disputas en el campo de la salud se inauguran en la década de los noventa con trabajos especialmente de tipo sociohistórico, orientados a comprender los procesos estructurales que configuran la política de salud en el país, los actores vinculados en la contienda y los recursos de poder que han tenido. Estos estudios mostraron la participación diferencial de actores nacionales e internacionales en la orientación de la política sanitaria, entre ellos profesionales de la salud-especialmente médicos- y trabajadores sindicalizados, y la mayor incidencia de actores internacionales en la orientación de la política en salud. Finalmente, encuentran que la política de salud ha institucionalizado un sistema de salud "fracturado", que reproduce internamente inequidades sociales. Sin embargo, estos estudios no se concentran en la comprensión de los procesos sociales vinculados a la configuración de actores colectivos por la salud.

En cuanto al periodo de la reforma del sistema de salud, la investigación encontró que hay una preocupación creciente por comprender los conflictos vinculados a la implementación de la reforma. En este sentido, se halló un giro de la mirada en el interior de este naciente campo de investigación. De un lado, las investigaciones que privilegian perspectivas teóricas estructuralistas del campo de la AC y los Ms, con enfoques predominantes de la escuela anglosajona como la estructura de oportunidades políticas (ЕOP) y la contienda política $(\mathrm{CP})$, pero también con incursión de un trabajo neomarxista. Estas investigaciones aportan una mirada procesual del campo de disputa, así como la identificación de actores, repertorios y recursos de poder. Los principales hallazgos se ubican en la comprensión de la política pública como un territorio de disputa, la historicidad de los actores ligados a esta, y el vínculo entre AC y reformas estructurales en salud. Sin embargo, adolecen de la pregunta por los procesos de configuración de actores colectivos, ya que se concentran en comprender los actores vinculados a la AC y la disputa de la política pública a nivel legislativo.

De otro lado, se encontró de manera clara un grupo más reciente de investigaciones que se preguntan por los procesos de constitución de actores colectivos. En este sentido, metodológicamente estos trabajos transitan de análisis de tipo documental hacia investigaciones cualitativas de tipo etnográfico, las cuales se preguntan por el sentido de la acción social para los actores vinculados. Utilizan marcos teóricos diferentes, como teorías de la resistencia o construccionistas. Sin embargo, los trabajos aún son muy heterogéneos y con excepción de dos de ellos, no se incursiona en los cuerpos teóricos de la Ac y los ms. Durante la investigación no se encontró ningún trabajo de revisión o balance sobre el tema, ni tampoco investigaciones empíricas de un alcance mayor.

En síntesis, se concluye que a pesar de los distintos esfuerzos investigativos, aún hay 
muchos vacíos para conocer y comprender los procesos de lucha y movilización social por la salud que se han desarrollado en el contexto de la implementación de la reforma. Asimismo, hay pocas aproximaciones específicas a este tema y los enfoques teóricos dominantes se pueden considerar estructuralistas. Reconociendo su aporte, habría que señalar, sin embargo, que no ubican como central el análisis de la configuración de actores colectivos. A lo anterior es necesario adicionar que una limitación de este estudio es no haber realizado un balance del tema a nivel internacional, especialmente en América Latina, región donde la conflictividad social se ha intensificado en los últimos años y se disputa alrededor de la universalidad de los sistemas de salud.

Se recomienda, de un lado, realizar un balance de este campo de investigación a nivel de América Latina, y de otro lado, profundizar esta línea de investigación en el país, dada la evidente conflictividad social generada por la disputa en salud y la creciente importancia del tema en la agenda académica nacional. Se llama la atención a la necesidad de investigaciones empíricas sobre AC, MS, actores y papeles dentro del Ms, entre otros.

Finalmente, no se puede olvidar que la salud, como parte de las necesidades básicas de los seres humanos, ha suscitado discusiones y debates, luchas y confrontaciones en diferentes lugares y épocas. Ante las múltiples vulneraciones del derecho a la salud producto de la Ley 100, es pertinente conocer los procesos de lucha y movilización social que intentan fracturar los discursos y las prácticas hegemónicas y comprender cómo se busca un lugar para la realización de la salud como un derecho humano fundamental. Para esto se debe ubicar, como asunto central, la mirada en los procesos de configuración de actores colectivos subalternos que disputan el campo sanitario, perspectiva de la AC y los Ms que aportan una comprensión enriquecida de cómo se ha ampliado la esfera pública en salud en Colombia.

\section{Referencias bibliográficas}

1. Múnera Ruiz L. Rupturas y continuidades. Poder y movimiento popular en Colombia 1968-1988. Bogotá: Iepri-Universidad Nacional de ColombiaCerec; 1998.

2. Borrero Y, Echeverry M. Luchas por la salud en Colombia. Una propuesta teórica para su análisis. Rev Gerenc Políti Salud. 2011; 10 (21): 61-82.

3. Archila M. Movimientos sociales, Estado y democracia en Colombia. Bogotá: ICANH, Universidad Nacional de Colombia.

4. Kornhauser W. Aspectos políticos de la sociedad de masas. Buenos Aires: Amorrortu; 1969.

5. Olson M. La lógica de la acción colectiva: bienes públicos y la teoría de los grupos. México: Noriega/ Limusa; 1992.

6. Tanaka M. Elementos para un análisis de los movimientos sociales. Análisis Político. 1995; 25 (2).

7. Galeano ME, Vélez O. Investigación cualitativa: Estados del arte. Informe de investigación Estrategias de investigación social cualitativa: el giro de la mirada. Medellín: La Carreta Editores; 2012.

8. Galeano M. Investigación documental: una estrategia no reactiva de investigación social. Estrategias de investigación social cualitativa: el giro de la mirada. La Carreta Editores; 2004. p. 113-44.

9. Archila M. Las luchas sociales en Colombia 19752000. Bogotá: Cinep; 2002.

10. Archila M. Idas y venidas vueltas y revueltas. Protestas sociales en Colombia. Bogotá: ICANH, Universidad Nacional de Colombia; 2003.

11. Escobar A, Pedroza A. Pacífico ¿desarrollo o diversidad? Estado, capital y movimientos sociales en el Pacífico colombiano. Bogotá: Cerec-Ecofondo; 1996.

12. Hernández E. Resistencia civil artesana de paz: experiencias indígenas, afrodescendientes y campesinas. Bogotá: Pontificia Universidad Javeriana; 2004.

13. Hernández Lara J. Formas de acción colectiva contra la guerra en el movimiento indígena del suroccidente colombiano. Acción política no-violenta una opción para Colombia. Bogotá: CEPI- Universidad del Rosario; 2005.

14. Ruíz CA. La rebelión de los límites (Quimeras y porvenir de derechos ante la opresión). Bogotá: Ediciones Desde Abajo; 2008.

15. Nieto López JR. Non armed civil resistance in Medellin. Voice and escape for the urban communities. Análisis Político. 2009; 22 (67): 37-59.

16. Bourdieu P, Wacquant L. Respuestas por una antropología reflexiva. México: Grijalbo; 1995.

17. Quevedo Vélez E, Hernandez Álvarez ME, Miranda Canal N. Ciencias médicas, Estado y salud en Colombia 1886-1957. Bogotá: Colciencias; 1993.

18. Hernández Álvarez ME. La salud fragmentada en Colombia 1910-1946. Bogotá: Unilibros; 2002. 
19. Hernández Álvarez ME. La fragmentación de la salud en Colombia y Argentina: una comparación sociopolítica, 1880-1950. Bogotá: Universidad Nacional de Colombia; 2004.

20. Hernández Álvarez ME. El debate de la Ley 100 de 1993 antes, durante y después. En Franco S, editor. La salud pública hoy: enfoques y dilemas contemporáneos en salud pública. Bogotá: Universidad Nacional de Colombia; 2003.

21. Gómez CA. Influencia de los grupos de interés y asociación en las reformas y los sistemas de salud. Rev Gerenc Políticas Salud. 2005; 4 (9): 62-80.

22. Organización Panamericana de la Salud. La ops y El Estado colombiano: 100 años de historia 19022002. Bogotá: ops; 2002.

23. Hernández Álvarez ME. El derecho a la salud en Colombia: Obstáculos estructurales para su realización. Rev Salud Pública. 2000; 2 (2): 121-44.

24. De Currea V. El derecho a la salud en Colombia, Diez años de frustraciones. Bogotá: Instituto Latinoamericano de Servicios Legales Alternativos, Ediciones Ántropos; 2003.

25. Arbeláez M. La protección constitucional del derecho a la salud: La jurisprudencia de la Corte Constitucional colombiana. Derecho Salud. 2006; 14 (2): 205-40.

26. Borrero Ramírez Y. La reforma del sector salud y los conflictos clasistas en Colombia: El caso de la Ley 100 [Tesis de grado para optar al título de Magíster en Sociología]. Cali: Universidad del Valle, Facultad de Ciencias Sociales y Económicas; 2008.

27. Uribe M. La contienda por la reforma del sistema de salud en Colombia (1990-2006) [Tesis de grado para optar al título de Doctora en Ciencia Social]. México, D.F: Colegio de México, Centro de Estudios Sociológicos; 2009.

28. Ocampo Morales DI. Perfil de la acción colectiva en salud motivada por la crisis hospitalaria en Colombia, 1994-2008 [Trabajo de grado para optar al título de Pregrado Administradora en Salud]. Medellín: Facultad Nacional de Salud Pública, Universidad de Antioquia; 2012.
29. Torres Tovar M. Acción social colectiva por el derecho a la salud en Colombia 1994-2010. El caso de Bogotá [Tesis de grado para optar al título de Magíster en Estudios Políticos]. Bogotá: Universidad Nacional de Colombia; 2011.

30. Morales Borrero C. Dinámica sociopolítica de la configuración de las políticas de salud sexual y reproductiva para jóvenes, Colombia 1992-2005 [Tesis de grado para optar al título de Doctora en Salud Pública]. Bogotá: Universidad Nacional de Colombia; 2010.

31. Urrego Mendoza Z. De protestas, violencias y otras fiebres tropicales. Aportes para una historia sociopolítica de la salud pública en Colombia, 1974-2004 [Tesis de grado para optar al título de Doctora en Salud Pública]. Bogotá: Universidad Nacional de Colombia; 2011.

32. Cárdenas Motta H. Hospital San Juan de Dios. Voces, pieles y resistencias. Bogotá: ATI; 2004.

33. Escobar C, De Roux F. Movimientos populares en Colombia 1970-1983. Los movimientos populares en América Latina, segunda edición. México: Siglo XXI Editores, Universidad de las Naciones Unidas; 2005.

34. Cotes Cantillo KP. Participación social en salud y democracia [Tesis de grado para optar al título de Magíster en Salud Pública]. Bogotá: Universidad Nacional de Colombia; 2006.

35. Echeverry E. El derecho a la salud en Medellín, Colombia desde la perspectiva de los usuarios, en el contexto de la reforma: 1990-2006 [Trabajo de investigación Doctorado en Ciencias en Salud Colectiva]. México: Universidad Autónoma Metropolitana Unidad Xochimilco; 2008.

36. Abadía Barrero CE, Oviedo Manrique DG, Martínez Parra AG. Movilización social por la defensa del derecho a la salud en Bogotá: esfuerzos locales y conexiones globales. Pre-Til. 2008; 6 (17): 46-63.

37. García Laíno L, Betancur Ossa N. Martes por la Salud: Un caso de luchas sociales en Medellín [Tesis de grado para optar al título de Pregrado Administrador en Salud]. Medellín: Universidad de Antioquia, Facultad Nacional de Salud Pública; 2013. 